\title{
Components and Analysis Method of Enterprise Resource Planning Requirements in Small and Medium Enterprises
}

\author{
Yousef Khaleel, Anmar Abuhamdah, Mutaz Abu Sara, Bassam Al-Tamimi \\ Department of Information Systems, College of Science and Computer Engineering, Taibah University, \\ Medina, Saudi Arabia
}

\begin{abstract}
Article Info
Article history:

Received Jun 16, 2015

Revised Nov 18, 2015

Accepted Dec 11, 2015

\section{Keyword:}

Analysis method Business processes ERP

SMEs

ABSTRACT

With the fast development of information technologies and enterprise software, Enterprise Resource Planning (ERP) systems are increasingly adopted by more small and medium enterprises (SMEs). Based on this trend, it is necessary to develop ERP systems in a manner that meets and fits the SMEs requirements and needs. This paper proposes conceptual components of ERP requirements that are required for generating ERP system functions. In addition, it proposes an ERP requirements analysis method for ERP system developments in order to produce the proper ERP system functions for SMEs. The advantage of this analysis method is that it is easy to analyze and integrate the special requirements of the ERP development for distinguishing a sub-sector of SMEs. In this paper, by analyzing the components of requirements and the relationship of the business process modelling, several basic concepts are given and the method of the process analysis and modelling is also expressed.
\end{abstract}

Copyright (C) 2016 Institute of Advanced Engineering and Science. All rights reserved.

\section{Corresponding Author:}

Yousef Khaleel,

College of Science and Computer Engineering, Taibah University,

PO box 344, Medina, KSA.

Tel: +962542032454

Email: ykhaleel@taibahu.edu.sa

\section{INTRODUCTION}

Enterprise Resource Planning (ERP) systems have long promised streamlined and efficient end-toend processes for enterprise. Several vendors (e.g. SAP, Microsoft, Oracle) offer ERP solutions (e.g. SAP $\mathrm{R} / 3$, Dynamics, Peoplesoft) for large and SMEs competing in various industries. In spite of the substantial promise of ERP systems, ERP implementation efforts have only seen mixed results. The lack of success in implementing ERP systems has been due to different reasons including projects being late or over budget, trouble during implementation, issues with the ERP systems configration, and problems with application integration [1].

Rolland and Prakash [2] noted two potential reasons that have not been highlighted in prior literature, including: (1) requirements analysis components, which include user requirements, system requirements, and business requirements. (2) The use (or the lack of use or inappropriate use) of systems development methodologies, which are used in conjunction with the component requirements to decide ToBe functional requirements (To-Be requirements are the ones to be satisfied in its future functioning). Whilst an ERP implementation attempt is generally perceived as an integration effort (to bind the ERP software to the enterprise's existing information systems), it essentially follows a lifecycle from beginning to end and is likely to gain benefit from the use of systems development methodologies. This research highlights the investigation of the requirements analysis components that are required for generating ERP functional requirements. In addition, this paper proposes a method that is able to use the identified requirements components in order to produce proper ERP system functions for SMEs. 


\section{CURRENT STATUS OF ERP SYSTEM IN SMES}

The technological issues, particularly business processes technologies, play an important role in the lack of ERP system adoption [4], [3]. These issues indicate that the complexity and non-compatibility of the off-the-shelf ERP systems have made them inappropriate for SMEs functions, and some of these functions were not used within SMEs area. Additionally, developing compatible In-house ERP systems with the business functions of the enterprise to fulfil its specific needs and requirements results in a high development cost and long implementation time [3], [5].

Based on extensive analysis for each of the issues above, it was found that the high cost and long implementation time of In-house ERP system development resulted from the customization and alignment needed. However, this high cost could be reduced by providing a customizing based on the sub-sector rather than on each enterprise individually. In other words, the enterprises in the same sub-sector have similar output products (activities) and are implicitly sharing similar business processes [6], [7]. Therefore, it can be deduced that those enterprises are qualified to share ERP system functions. Hence, in order to derive the specification for successful ERP adoption, it is proposed to develop customized ERP systems that meet the needs of those sub-sectors, each separately. According to Khaleel and Sulaiman [8] and Khaleel et al. [3], it is not necessary for the SMEs to adopt the ERP systems designed for large scale companies. Instead they can adopt the customized ERP systems that cater to the specific business needs of each sub-sector.

This research proposed a requirements analysis components and system analysis method for ERP systems to produce the intended ERP system functions for each distinguished sub-sector of SMEs.

\section{CONCEPTUAL COMPONENTS OF ERP REQUIREMENTS}

The purpose of conceptual components is to generate ERP functional requirements, based on improving the current business processes and aligning ERP system functions. In order to produce the proper ERP system requirements fitt the targeted business, this paper proposed conceptual components of ERP which is utilized three components that had a vital role in generating compatible ERP functional requirements. As shown in Figure 1, each component represents a type of requirement.

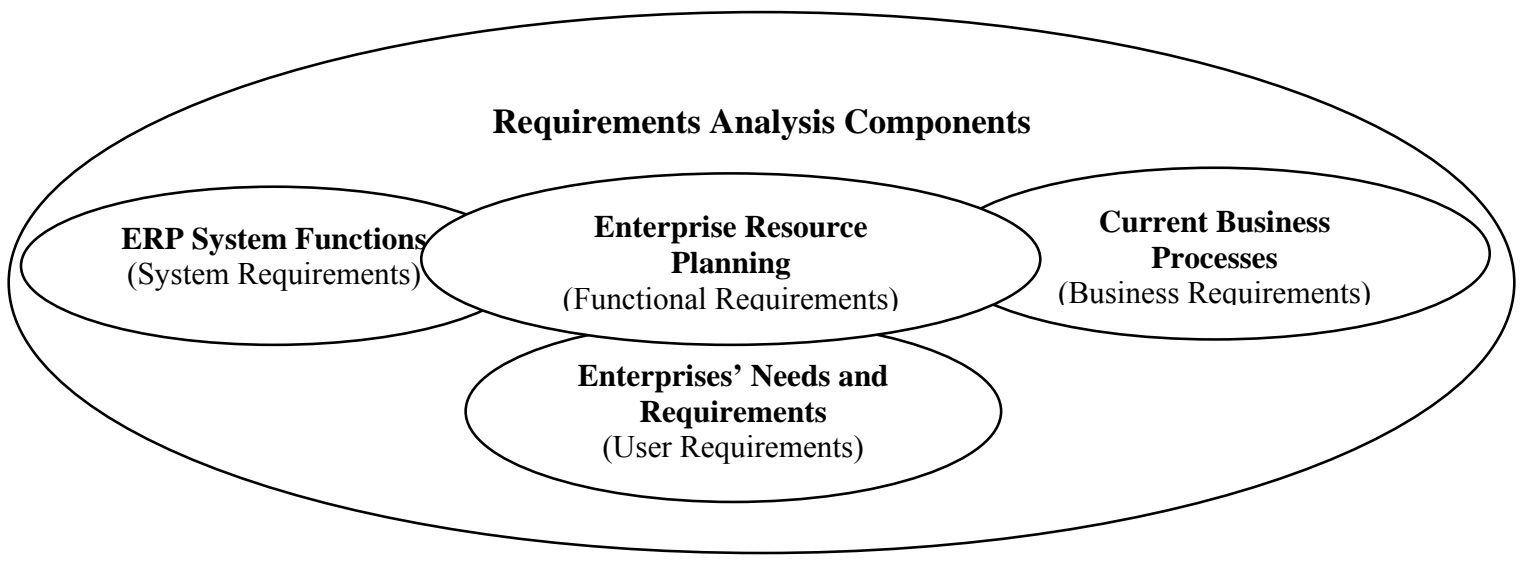

Figure 1. Conceptual components of ERP requirements analysis

\subsection{ERP System Functions Based Requirements (System Requirements)}

ERP software is made up of many software modules. Each ERP software module mimics a major functional area of an organization. A better understanding of ERP modules components and functions will directly help in the selection of appropriate ERP functions that meet the organization needs and requirements [9].

The previous developers of ERP systems have suggested that, for this technology, we need to understand the scope of the ERP modules and have a basic knowledge of how they integrate and work together. The ERP system functions and the relationships between each of these functions and the rest of a total enterprise integration system are included in the system requirements. 


\subsection{SMEs Sub-Sector Needs and Requirements (User Requirements)}

In order to realize and work with the issues in developing an ERP in SMEs, the ERP developers and vendors should understand the needs and main characteristics of SMEs, assuring the success of the ERP implementation [10]. It is vital and compulsory to understand the way in which the SMEs differ from large organizations. By understanding the characteristic of SMEs in terms of ERP implementation, it can lead to choosing the right ERP system functions for SMEs [11]. The needs and the current situation of SMEs in terms of ERP could be identified by conducting one of the requirements collection techniques such as interviews, questionnaires, or observations.

\subsection{Current Business Process Based Requirements (Business Requirements)}

Identifying a current business process well will give a clear and precise idea of As-Is business process and use of enterprise assets, capacity utilization, and the different processes currently in place, to produce the outputs required. It can also provide insight into appropriate ERP functions that their implementations are inexpensive and efficient to reduce the overall cost base without any impact on ERP system quality and users satisfaction [12]. The significance of defining current business processes lies in the need for aligning and understanding various business units and geographies, how things currently operate, and to set the base by which these process can be developed As- Is without any changes and the process needed to re-engineer, modelled to meet the ERP functions.

Marnewick and Labuschagne [13] have proposed ERP model consists of four components that are implemented through amethodology. Figure 2 illustrates the integration between the components.

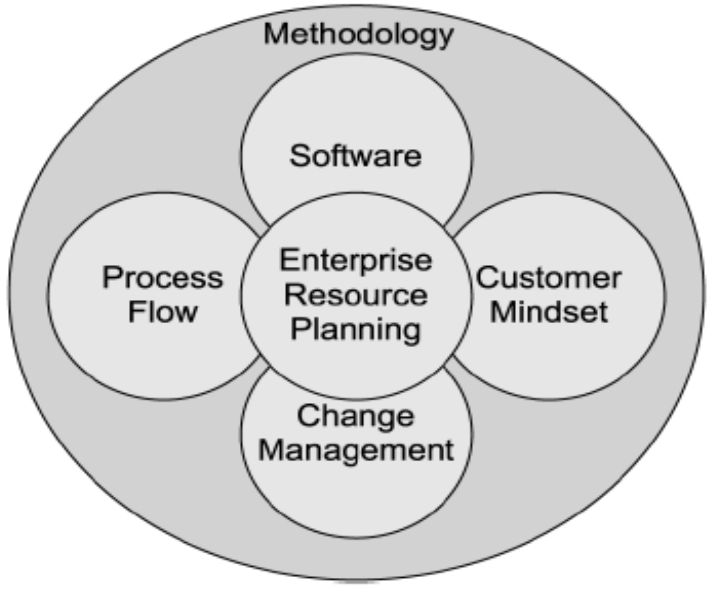

Figure 2. ERP components model

A clear mapping results, as can be seen in Table 1, when the prposed conceptual components of ERP requirements analysis is compared to ERP model.

Table 1. Applying the ERP model to conceptual components of ERP requirements analysis

\begin{tabular}{cc}
\hline ERP model & Conceptual components of ERP requirements analysis \\
\hline Customer mindset & Enterprises' Needs and Requirements (User Requirements) \\
Software & ERP System Functions (System Requiremen) \\
Change management and Process flow & Current Business Processes (Business Requirements) \\
\hline
\end{tabular}

\section{ERP REQUIREMENTS ANALYSIS METHOD}

ERP requirements analysis method has been proposed using the three identified components of ERP requirements (ERP system functions, current business processes, and enterprises' needs and requirements); this method aims to generate ERP system's functionality and necessary alternative business process that achieve the desired objectives of the ERP system. The Figure 3 briefly describes the proposed analysis method of ERP functional requirements. 


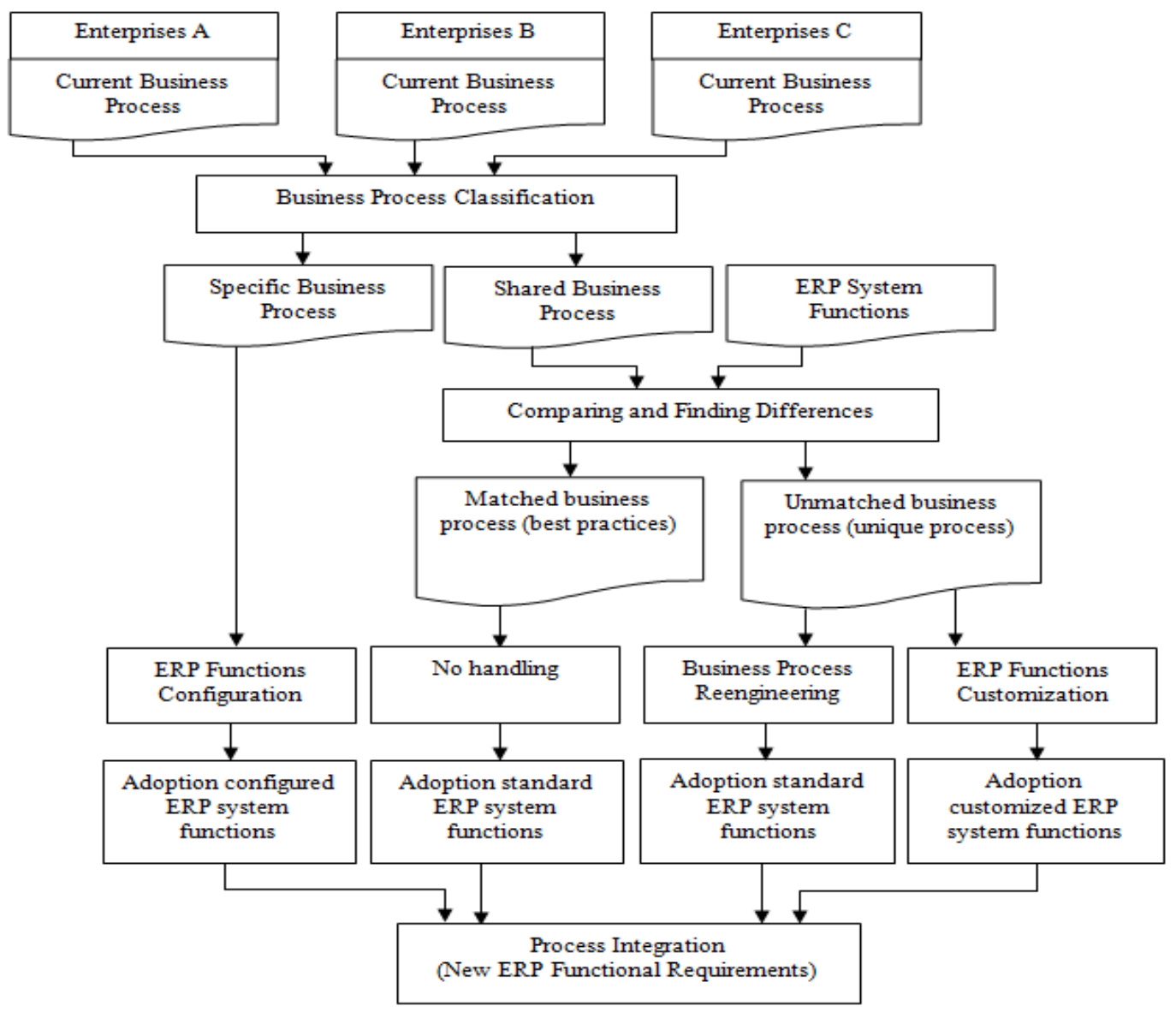

Figure 3. ERP requirements analysis method

The proposed method is a systematic approach, which helps to identify the new ERP system functions, through which a proper combination among the three requirements components is ensured, as illustrated in Figure 1. Furthermore, the analysis method is built on a theory that integrates the identified requirements to achieve the declared objective of the ERP system.

First of all, the current business processes of the three selected enterprises (could be more than three enterprises) were compared with each other to find the similarities and differences in business process within these enterprises. The different business processes within the enterprises were analyzed using a configuration technique. The configuration was used to maintain the privacy of each enterprise's process and characteristics. Secondly, the shared enterprises' business process was compared with the ERP system functions to find the differences [14]. The output of this step is to find out two kinds of business processes, which are matched business process with ERP functions and unmatched business processes with ERP function.

The ERP system was implemented to the current business process that has matched with the ERP functions without any adjustment in both of ERP system functions and current business process this is due to the harmony between the ERP function and business process. In this case the business processes are called best practices processes. The ERP customization and business process reengineering technique have been identified in order to analyze the unmatched business processes. Both identified requirements modelling techniques were chosen based on the needs of the enterprises.

Finally, all of the identified ERP system requirements were matched and integrated to provide new ERP system functions (functional requirements) to achieve the enterprises' needs and requirements. The following is a brief discussion on each of the ERP functional requirements analysis steps.

\subsection{Business Process Classification (Step One)}

This step aims to classify the business processes, as it helps to define the suitable analysis techniques for each business process. Based on analyzing the document-based research for current business 
process conducted earlier, the results of classifications have showed two types of business processes as follows: (1) Shared business processes which intended to the business processes were found to be similar, shared within all enterprises in the selected SMEs sub-sector. (2) Specific business processes: which intended to the business processes were found to be exclusive and limited to specific enterprises. Therefore, such business processes cannot be standardized for other enterprises, including the enterprises within the selected sub-sector.

\subsection{Comparison of Shared Business Processes (Step Two)}

The targeted business processes in this step of analysis are the processes that are similar across all enterprises within the selected sub-sector. Developing suitable ERP system functions does not inevitably mean redefining an organization's business processes. The ERP system can be used to improve the existing business process; nevertheless, an outright re-engineering might be precisely needed in some cases, when the current business processes do not match with ERP functions [15], [16], these business processes are as called unique processes [16]. Based on the above the current business process (As-Is business process) of the selected enterprises was compared with the ERP system components and functions as defined in standard ERP system functions, to identify which business processes matched or did not match with ERP functions. The output of the comparison step is two types of shared business process as following: (1) Matched business process (best practice) which is business processes that are matched with the ERP system functions; these business processes are adapted to an ERP system without modification. (2) Unmatched business process (unique process) these are processes that did not match with the functions of the ERP system. In this case, some business processes need to be enhanced and optimized in order to meet the standard ERP modules (Business Processes Reengineering). While in the other analysis technique, the unmatched business processes remain unchanged. Instead of re-designing these processes, the ERP system functions are customized to fit these business processes.

The following steps explain the actions taken for the matched and unmatched business processes, although there are no specific modifications to be imposed for the matched business processes.

\subsection{Matched Business Processes Analysis (Step Three)}

This group consists of the process, which has matched with best practices standards of ERP. These processes have been adapted to ERP standard functions, without any modification in both types of process and ERP standard functions.

\subsection{Unmatched Business Processes (Unique Processes) Analysis and Improvements (Step Four)}

The enterprise enables to identify the ERP functions that satisfies stated unique business processes that do not necessarily match any predefined "best practice" solution, some of unique business processes that are not satisfied by the system [16]. For this study we have proposed two techniques of analysis, in order to match the specifications of the unique business process enterprises with ERP system capabilities.

The first adopted technique is customization of ERP modules. The customization strategy concentrates on the backing of a couple of basic business processes, which includes a few business units. The introductory customization of the ERP system is constrained to functionality to fit these business processes without any improvements or modification on these current processes. The second technique is business processes redesign. This analysis technique is applied to the unique business processes that are not satisfied by the ERP system, and cannot customize the entire ERP system functions, to match with it; in this case, we have to redesign these processes, in order to match with ERP best practices. Both analysis techniques used to define the new ERP system functions (functional requirements) were briefly described in next analysis step.

\subsubsection{ERP Functions Customization}

The creating of an ERP system includes a procedure of customizing the generic package and adjusting it to the particular needs of the enterprise [14]. Improving the system's functionality through customization, while undesirable, is some of the time obliged [17]. The business process alignment is influenced by different environmental perspectives, for example, existing information systems preceding the ERP usage and organizational culture [16].

This study is motivated by the necessitate to provide support for the alignment process in enterprises, which business processes include unique processes (unmatched with the ERP systems functions). Such enterprises do not necessarily wish to frequently standardize all their processes due to ERP implementation. Common tools that support the alignment process refer to predefined "best practice" models [16], and therefore do not support the needs of these enterprises. Preserving the unique processes of enterprises may require these enterprises to make software customizations in the ERP system and exploit the benefits of customizations in terms of fitting the enterprises' business processes, , avoiding the high cost of a 
new process design or "best practice" model while totally ignoring the existing unique process [17]. Nevertheless, the functionality of ERP system in most cases is sufficiently rich and fit of supporting business processes that are not included in the "best practice" solutions [16]. Such a methodology matches Characteristics of the enterprise requirements with a model, indicating the ERP system abilities. This requires the model of the ERP system abilities, to demonstrate the whole extent of options available in the ERP system, which empowers matching with the enterprise requirements.

The need for applying custom ERP modules has emerged because of the benefits offered by the customization, these benefits meet the SMEs needs as follows: (1) developing uncomplicated ERP functions that fit the organization's business process, (2) compatible and adequate ERP functions, and (3) low cost and time of implementation, where that customization will shorten the business processes re-design as much as possible, which require time and high cost [7].

\subsubsection{Business Process Alignment and Redesign}

Implementing an ERP system does not necessarily mean redefining an organization's business processes. The ERP System can be used to enhance and optimize the existing business process [14], [15], [18]; however, an outright re-engineering might be needed in some cases. Precisely, when the current business process does not match with ERP functions, the business process is unique to the enterprise [14]. Redesigning the unique business process may require these enterprises to take the risks of long implementation time and high cost for change processes [18].

Due to the risks of the redesign practice, redesign is considered as a last choice to be taken, in order to match the unique business process of enterprises with a standard ERP function. Therefore, the business process redesign is performed for unique business processes that do not meet the standard ERP system. As it is also impossible to customize the entire ERP system functions to match these unique processes.

\subsection{Specific Business Processes Analysis (Step Five)}

Specific business processes are the business processes that vary among the enterprises and cannot be standardized among the selected enterprises within specific SMEs sub-sector. The configuration technique has been selected to analyze these processes.

\subsubsection{ERP Functions Configuration}

Configuring an ERP system is largely a matter of balancing the way the customer wants the system to work with the way it was designed to work. The ERP systems typically build many changeable parameters that modify the system's operation. For example, an organization can select the period of time for running master schedule computing, which they can run daily, weekly, or monthly [19].

On the other hand, a business may configure a system to its needs by selecting appropriate system components and by setting parameters that allow the organization to modify the system within the boundaries set by the developers of the enterprise application [19].

Based on the above discussion, the ERP configuration is adopted to develop flexible and adjustable ERP system functions, as it allows each enterprise to set the ERP functions so that it fits its specific business process, which is different from other enterprises. In addition, the configuration has allowed the enterprises to choose the needed ERP functions and ignore unneeded functions.

\section{CREATE NEW ERP SYSTEM REQUIREMENTS DEFINITION}

As all requirements have been identified, they were then studied and reviewed before they were put into a structure according to their inter-relationships among the requirements gathered. The requirements were reviewed to eliminate any redundancies.

The system requirements can be either functional or non-functional in nature. A functional requirement relates directly to a process the system has to perform or information it needs to contain. An information-oriented functional requirement would be that, the system must include [13]. Functional requirements flow directly into the design and modelling process (use cases, process models, data models), because they define the functions that the system needs to have. Non-functional requirements refer to behavioural properties that the system must have, such as performance and usability.

\section{CONCLUSION AND FUTURE WORK}

We presented a methodology which bolsters the arrangement of ERP system functions to customer requirements. It comprises of a process method to show the way ERP requirements match SMEs requirements. Specifically, this method (systematic approach) consist of abstraction step to abstract from 
ERP requirement analysis components thereby (a) establishing a post-traceability link between ERP requirements and functionality and (b) providing an intentional view of what the ERP system can do. The latter is necessary to support strategic decisions about the parameterization of the ERP system to fit the enterprises business policy. The previous provides a clear way to select the ERP functionality together with its variants compliant to the matched requirements. The requirements matching process are principally ERP driven. This is the way by which we can reduce the number of deviations from the ERP functionality in a given installation and also advantage from the full power of the ERP functionality.

The next step in our work is the application of the approach to a large scale industrial project. Also, we expect to lay down guidelines for applying the abstraction of functionality to tentative design.

\section{ACKNOWLEDGEMENTS}

We gratefully acknowledge the assistance of professor Riza Suliman. This research is supported by the Deanship of Scientific Research in Taibah University, KSA.

\section{REFERENCES}

[1] Jeyaraj, A., \& Sethi, V. (2008). Traditional and modern methodologies in ERP systems implementation using Microsoft dynamics-GP. Proceedings of the Southern Association for Information Systems Conference. Richmond, USA. 2008.

[2] Rolland, C., \& Prakash, N. (2001). Matching ERP system functionality to customer requirements. Proceedings Fifth IEEE International Symposium on Requirements Engineering. Toronto, Ont. 2001; 5: 66-75.

[3] Khaleel, Y., Sulaiman, R., Ali, N. M., \& Baharuddin, M. S. Analysis of Enterprise Resource Planning System (ERP) in Small and Medium Enterprises (SME) of Malaysian Manufacturing Sectors: Current Status and Practices. Jurnal Teknologi Maklumat \& Multimedia. 2011; 10(1): 13-20.

[4] Sankar, C., \& Rau, K. Technical Issues in Implementing ERP Systems. Implementation Strategies for SAP R/3 in a Multinational Organization: Lessons from a Real-World Case Study. Hershey, PA: CyberTech Publishing. 2006; 105-137. doi:10.4018/978-1-59140-776-8.ch005

[5] Huin, S. F. Managing deployment of ERP systems in SMEs using multi-agents. International Journal of Project Management. 2004; 22(6): 511-517.

[6] Poba-Nzaou, P., Raymond, L., \& Fabi, B. Adoption and risk of ERP systems in manufacturing SMEs: a positivist case study. Business Process Management Journal. 2008; 14(4): 530-550.

[7] Raymond, L., \& Uwizeyemungu, S. A profile of ERP adoption in manufacturing SMEs. Journal of Enterprise Information Management. 2007; 20(4): 487-502.

[8] Khaleel, Y., \& Sulaiman, R. A system development methodology for ERP system in SMEs of Malaysian manufacturing sectors. Journal of Theoretical \& Applied Information Technology. 2013; 47(2).

[9] Frazee, K., \& Khan, M. R. ERP implementation for corporate growth and sustainability. International Journal of Business and Social Sciences. 2012; 3(3): 74-82.

[10] Deep, A., Guttridge, P., Dani, S., \& Burns, N. Investigating factors affecting ERP selection in made-to-order SME sector. Journal of Manufacturing Technology Management. 2008; 19(4): 430-446.

[11] Bernroider, E., \& Koch, S. ERP selection process in midsize and large organizations. Business Process Management Journal. 2001; 7(3): 251-257.

[12] Esteves, J., Pastor-Collado, J., \& Casanovas, J. Monitoring business process redesign in ERP implementation projects. Americas Conference on Information Systems (AMCIS), Dallas, 2002. 12-31.

[13] C.L. Phillips and H.T. Nagle, Digital control system analysis and design, 4 ed.: Prentice Hall Press, 2007.

[14] Štemberger, M.I., \& Kovačič, A. The role of business process modelling in ERP implementation projects. Proceedings of the Tenth International Conference on Computer Modeling and Simulation (UKSIM), Washington, USA, 2008. 260-265.

[15] Eseyin, K. (2006, 11 Octoper 2010). ERP Implementation and Business Process Re-engineering. Available: $\mathrm{http} / / /$ it.toolbox.com/blogs/sap-library/erp-implementation-and-business-process-reengineering-11537

[16] Soffer, P., Golany, B., \& Dori, D. ERP modeling: a comprehensive approach. Information systems. 2003; 28(6): 673-690.

[17] Light, B. The maintenance implications of the customization of ERP software. Journal of software maintenance and evolution: research and practice. 2001; 13(6): 415-429.

[18] Panayiotou, N.A., Gayialis, S.P., \& Evangelopoulos, N. E. Integrating business process modelling and ERP role engineering. International Journal of Business Information Systems. 2011; 8(1): 66-86.

[19] Leitner, A., \& Kreiner, C. Managing ERP configuration variants: an experience report. In Proceedings of the 2010 Workshop on Knowledge-Oriented Product Line Engineering (KOPLE). New York, USA. 2010. 


\section{BIOGRAPHIES OF AUTHORS}

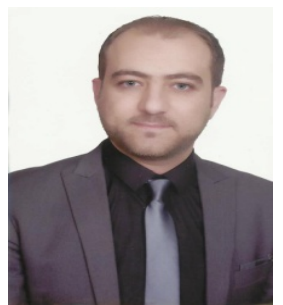

Yousef Khaleel received the B.Sc (Computer Information System) from Mu'tah University for in 2006. He received the M.Sc (Information Technology) from Universiti Utara Malaysia in 2008 After doing his Phd in Universiti Kebangsaan (UKM) Malaysia from 2013) with customizing Enterprise Resource Planning (ERP) System Modules for SMEs. His research interest includes system modelling, systems integration, software engineering. He work as assistant professor from 2013 till now at Taibah university.

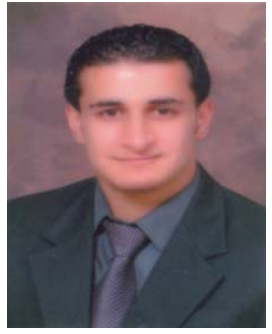

Anmar Abuhamdah received the BSc. degree in Computer Science from Princess Sumaya University for Technology (PSUT), Jordan in 2003. He received the Master degree in Science (Intelligent System) from Universiti Utara Malaysia (UUM), Malaysia in 2006. After that, he works as a lecturer in Almjd Quality, Malaysia. He received his PhD in Computer Science from Universiti Kebangsaan Malaysia (UKM), Malaysia in 2011. He works at Jerash University, Jordan from 2011 to 2012 as assistant professor. He is currently (2015) working as assistant professor in the College of Science and Computer Engineering, Taibah University, KSA from 2012. His research interest includes Timetabling scheduling, Real time system, Optimization, and Software development.

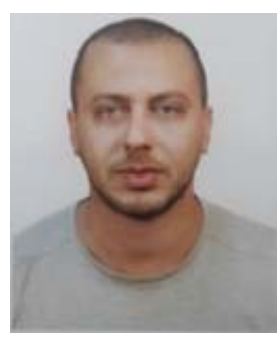

Mutaz Abu Sara received the B.Sc from Saint Petersburg Electrotechnical University in 2004. He received the Master of Science (Database Systems) from Saint Petersburg Electrotechnical University in 2006. After working as programmer at BiSoft Company in Saint Petersburg. After doing Phd in Saint Petersburg Electrotechnical University from 2007) with Research and Development of Integrated Database Circuit Components for CAD Schematic, His research interest includes Database Systems, Real Time System, Effectiveness of Design. He works as assistant professor from 2011 till now at Taibah University.

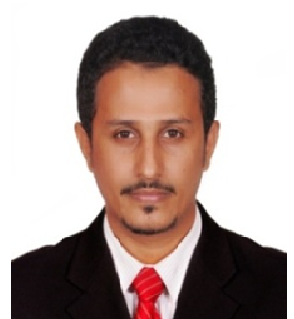

Bassam Naji AL-Tamimi received the BSc. degree in Computer Science from University of Science and Technology, Yemen in June 2005 and Master degree in Distributed Computing and Networks from Universiti Sains Malaysia (USM), Pulau Penang, Malaysia in 2009. He received his PhD in Network Security from Universiti Sains Malaysia (USM), in 2013. Since September 2014, he has been an assistant professor in the College of Science and Computer Engineering, Taibah University, KSA. His main research interests include Computer Networks, Information Security, Wireless \& Mobile Communications, Cloud computing infrastructure and services, Network Performance and Measurements. 